\title{
Deployment of a Forward Medical Post to Provide Medical Support in a Purpose-built Dormitory during the COVID-19 Pandemic
}

\section{Dear Editor,}

The first case of coronavirus disease 2019 (COVID-19) was reported in Singapore on 23 January $2020,{ }^{1}$ with the subsequent increase in cases within purpose-built dormitories (PBDs) in April 2020 leading to the introduction of control measures to care for migrant workers (MWs) residing in PBDs.

PBDs are licensed under the Foreign Employee Dormitories Act (FEDA). ${ }^{2}$ Each PBD houses MWs who share communal facilities. Mandatory requirements under FEDA include designated isolation rooms and pandemic contingency plans. However, most PBDs do not have a medical clinic on-site, necessitating MWs to visit community clinics for healthcare. Within the pandemic setting, this is not possible following need to isolate the dormitories and limit movement of the MWs. Hence, forward medical posts (FMPs) were deployed.

This article describes how the Singapore Armed Forces (SAF) FMP supported public health efforts at one of the largest PBDs, serving a MW population of approximately 13,000. The PBD was declared an isolation area under the Infectious Diseases Act on 5 April $2020^{3}$ to curb transmission within the dormitory and community. While the FMP worked alongside other civilian counterparts in an inter-agency team caring for the MWs, this article focuses on the SAF's ground perspectives and lessons learnt.

From 7 to 28 April 2020, the SAF FMP was deployed with the mission of (1) providing early identification and containment of COVID-19 infections, and (2) providing primary healthcare for $\mathrm{MW}$ residents ${ }^{3}$ to assist with national public health measures. ${ }^{4}$ Teams comprised 1 medical officer (MO), 1 military medical expert (MME) who is nursing- or paramedic-trained, and 5 medics for every 5000 occupants. The FMP was equipped with medical supplies for acute medical conditions, with surge capacity for acute respiratory infections (ARIs).

A taskforce headquarters was established to provide central coordination for all PBDs, and to establish the public health strategy in consultation with the Ministry of Health. The overall mandate, roles and responsibilities of the FMP were clearly defined by the medical cell in the taskforce headquarters. Through daily reviews, processes were refined to meet operational needs. One example was the development of standardised clerking sheets in Chinese, Malay, Tamil, Telugu and Bengali (Fig. 1). This helped to overcome language barriers, and provide a simplified template for clinical records. Data was consolidated and analysed on the backend, so that frontline staff could focus on healthcare delivery.

The deployment concept mirrored a primary healthcare clinic. Additionally, based on past humanitarian assistance and disaster relief (HADR) experience, distinct operating zones and lanes for human traffic were identified to reduce congestion (Fig. 2). Emergency cases were conveyed to tertiary healthcare institutions via ambulance, while cases that fulfilled case suspect criteria were conveyed to either swab isolation facilities (SIF) or restructured hospitals for further management.

Alongside clinical duties, the FMP was also responsible for the public health needs in the PBD. A senior medical representative with the role of public health officer (PHO) was embedded within the operational structure of the

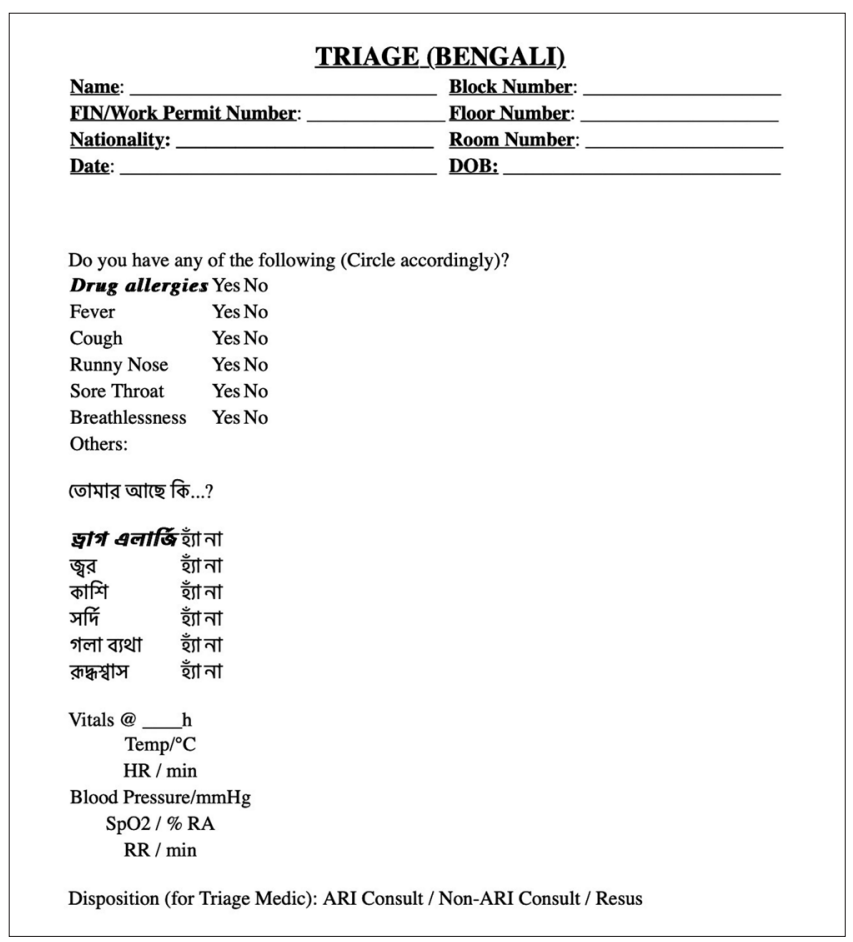

Fig. 1. Example of a clerking sheet in English and Bengali. 


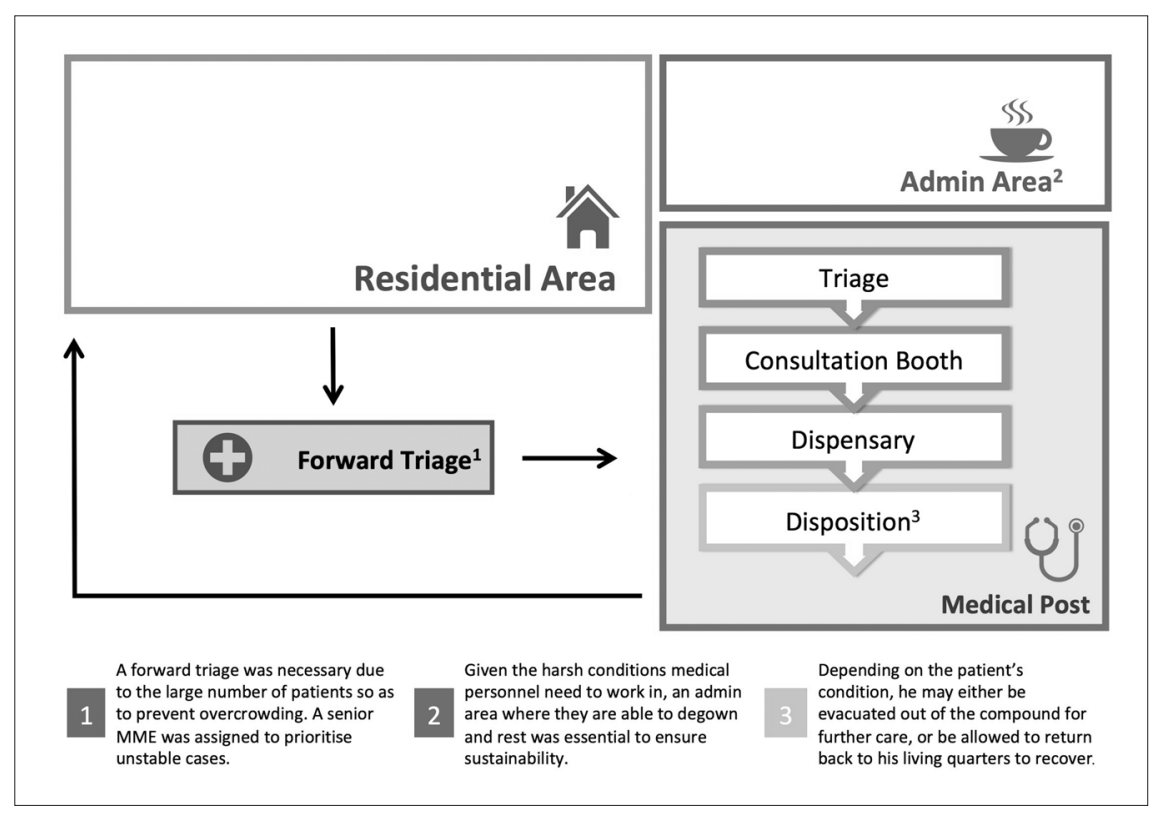

Fig. 2. Operating zones and identified lanes for human traffic to reduce congestion.

PBD. The PHO tailored plans to locational context, and provided advice through MW outreach campaigns such as education on good hygiene practices, physical distancing and mental wellness. To aid dissemination, the medical team and stakeholders identified approaches suited to the MW population, which included social media, public announcements and appointing dormitory ambassadors.

Medical care for the MWs spanned office hours during initial phases. Thereafter, telemedicine was made available to MWs to extend medical care beyond the FMP operational timings.

Active surveillance was performed to identify patients with severe disease $e^{5}$ early and prevent deterioration. For example, pulse oximeters were issued to MWs for selfmonitoring. Videos and posters were also promulgated so that MWs knew how to use the equipment, and identify abnormal parameters including oxygen saturation, heart rate and temperature.

A total of 1,942 patients were seen during the deployment, with an average of 88 patients per day. Among these, 1,550 were ARI cases, while the remaining were a spectrum of non-ARI acute illnesses and chronic medical conditions. Approximately $5-10 \%$ of patients needed evacuation to a tertiary hospital for further care. A parallel effort also took place to swab all close contacts within the dormitory. A total of 2,386 COVID-19 cases were diagnosed, including ARIs. Patients who needed more urgent care, and patients who displayed ARI features were prioritised for evacuation to appropriate facilities.

The medical team applied experience from HADR missions $^{6}$ to plan the medical support for this deployment.
The military's value lies in its ability to swiftly organise manpower, coordinate logistics, and adapt to volatile conditions. The planning considerations from HADR missions ${ }^{7}$ formed a framework, through which the authors would like to share 4 insights.

Firstly, caring for the people who drive HADR missions involves ensuring proper work-rest cycles and the welfare of personnel. In the pandemic setting, the leadership also focused on adopting a zero-tolerance policy for healthcare worker (HCW) infections, in relieving anxiety for HCWs to focus on the mission. Multiple safeguards were instituted. The area of operations was divided into 'clean' and 'contaminated' sectors, and personal protective equipment (PPE) was emphasised for all HCWs before entering contaminated sectors, through a 3-pronged system. First, alongside individual ownership, a buddy system reinforced mutual accountability. Second, direct supervision by MOs and MMEs was mandated for all donning and doffing of PPE. Third, audits by appointed safety advocates were implemented to identify safety breaches.

The FMPs had to be deployed in open areas and HCWs donned full PPE for extended periods, exacerbating risk of heat injuries and attrition ${ }^{8}$ in Singapore's hot and humid climate. To address this, work-rest cycles were strictly adhered, and an administrative rest area was designated. At the individual level, the deployment of military personnel who are heat acclimatised minimised potential heat injury.

At the end of the deployment, no HCWs were infected with COVID-19, or suffered from heat injuries. The authors believe that these safeguards allowed the HCWs 
to focus on their deployment, and played a significant role in realising a successful mission.

Secondly, components synonymous with military operations were implemented, including fixed daily routines, reporting cycles and a command hierarchy. These fundamentals were employed to build psychological self-discipline and team rhythm. Given inevitability of situational uncertainties, quick-fire decisions were frequently made, compelling ground leaders to understand overall intent and practise situational adaptation. While this principle may be an antipathy to doctrines, this was a concept adopted from the military principle of mission command. ${ }^{9}$

Thirdly, cooperation with non-governmental organisations (NGOs) and partners is crucial in disaster relief operations. This may include leaders and officials during HADR missions. For this deployment, strong rapport was built between the dormitory management and MWs, which facilitated management of large volumes of patients during clinic hours, and monitoring of MWs' follow-up care and isolation. NGO partners and formal MW ambassadors were also embedded within the medical team. This helped to bridge the communications gap, strengthen physician-patient relationships and provide a psychologically safe environment for MWs.

Lastly, medical support during the pandemic needs to be sustained beyond usual HADR missions due to the potential for exponential increase in disease numbers, and subsequent impact on the national healthcare if support is lifted. To this end, civil-military partnerships and early co-opting of primary healthcare teams from public regional healthcare systems was important to ensure continuous medical support for subsequent PBDs.

MW empowerment for self-care continues to be important. MWs were engaged on points essential to infection control including promotion of health seeking behaviour, basic hygiene and physical distancing. The authors recognised that MWs who have been isolated are at increased risk of mental health issues due to lack of social support and uncertainty regarding repatriation. ${ }^{10}$ NGOs provided augmentation in areas such as production of communication materials and counselling services for mental wellness. This parallel is witnessed in HADR, where NGOs and stakeholder involvement are required to ensure the return to normalcy. ${ }^{11}$

The authors believe that the successful deployment of the FMP in challenging circumstances hinged on alignment of team objectives, establishing structures, continuous reviews and adaptive learning. While the experience is a slice of the national public health strategy, the lessons learnt may serve as reference for future medical planners and their missions.

\section{Acknowledgement}

The authors would like to thank all members of the SAF medical team for their invaluable contributions and sacrifices.

\section{REFERENCES}

1. Ministry of Health, Singapore. Confirmed Imported Case of Novel Coronavirus Infection in Singapore; Multi-Ministry Taskforce Ramps Up Precautionary Measures. Available at: https://www.moh. gov.sg/news-highlights/details/confirmed-imported-case-of-novelcoronavirus-infection-in-singapore-multi-ministry-taskforce-rampsup-precautionary-measures. Accessed on 31 July 2020.

2. Foreign Employee Dormitories Act 2015 (No. 3 of 2015). Available at: https:/www.ilo.org/dyn/natlex/docs/ELECTRONIC/100522/1206 13/F-1038074821/SGP100522.pdf. Accessed on 31 July 2020.

3. Ministry of Health, Singapore. Additional Measures To Minimise Further Spread Of Covid-19 Within Foreign Worker Dormitories. Available at: https:/www.moh.gov.sg/news-highlights/details/ additional-measures-to-minimise-further-spread-of-covid-19-withinforeign-worker-dormitories. Accessed on 31 July 2020.

4. Hsu LY, Chia PY, Lim JF. The Novel Coronavirus (SARS-CoV-2) Epidemic. Ann Acad Med Singap 2020;49:105-7.

5. Wu Z, McGoogan J. Characteristics of and Important Lessons From the Coronavirus Disease 2019 (COVID-19) Outbreak in China: Summary of a Report of 72314 Cases From the Chinese Center for Disease Control and Prevention. JAMA 2020;323:1239-42.

6. Danguy des Déserts M, Mathais Q, Luft A, et al. Conception and deployment of a 30-bed field military intensive care hospital in Eastern France during the 2020 COVID-19 pandemic. Anaesth Crit Care Pain Med 2020;39:361-2.

7. Ho ML, Lim JZ, Tan MZ, et al. Humanitarian Assistance and Disaster Relief mission by a tripartite medical team led by the Singapore Armed Forces after the 2015 Nepal earthquake. Singapore Med J 2016;57:426-31.

8. Loibner M, Hagauer S, Schwantzer G, et al. Limiting factors for wearing personal protective equipment (PPE) in a health care environment evaluated in a randomised study. PLoS One 2019; 14:e0210775.

9. Pearce AP, Naumann DN, O'Reilly D. Mission command: applying principles of military leadership to the SARS-CoV-2 (COVID-19) crisis. BMJ Mil Health 2020;bmjmilitary-2020-001485.

10. Ho CS, Chee CY, Ho RC. Mental Health Strategies to Combat the Psychological Impact of COVID-19 Beyond Paranoia and Panic. Ann Acad Med Singap 2020;49:155-60.

11. Nishiyama Y. Disaster Relief Activities of the Japan Self-Defense Force Following the Great East Japan Earthquake. Disaster Med Public Health Prep 2014;8:194-8.

Guan Lin Lee, ${ }^{1} M B B S$, Wei Ting Lee, ${ }^{1} M B B S, F R C S E$, Wai Leong $\underline{\text { Kok }},{ }^{1} M B B S$, FAMS

${ }^{1}$ Headquarters Army Medical Services, Singapore Armed Forces, Singapore

Address for Correspondence: Dr Guan Lin Lee, Headquarters Army Medical Services, Singapore Armed Forces, 701 Transit Road, Singapore 778910. Email: Lee_Guan_Lin@defence.gov.sg; leeguanlin@gmail.com 